\title{
The novel circSLC6A6/miR-1265/C2CD4A axis promotes colorectal cancer growth by suppressing p53 signaling pathway
}

\author{
Zeyin Rong ${ }^{1 \dagger}$, Zai Luo ${ }^{1 \dagger}$, Zhongmao Fu ${ }^{1 \dagger}$, Pengshan Zhang ${ }^{1 \dagger}$, Tengfei $\mathrm{Li}^{1}$, Jianming Zhang ${ }^{1}$, Zhonglin Zhu',
} Zhilong Yu' ${ }^{1}$ Qi Li ${ }^{2,3}$, Zhengjun Qiu ${ }^{1}$ and Chen Huang ${ }^{1 *}$

\begin{abstract}
Background: Colorectal cancer (CRC) is one of the most frequent malignancy and a leading cause of cancer-related deaths. Therefore, further researches are required to identify novel and more effective diagnoses and to identify molecular targets in treatment of CRC.

Methods: C2CD4A expression in CRC tissues and cell lines was detected by qRT-PCR and western blot. The biological functions of C2CD4A were performed both in vitro and in vivo. Western blot, CDNA array, IP-MS, Co-immunoprecipitation assay, and Ubiquitination assay were used to analyze the interaction between C2CD4A and p53. Bioinformatics analysis, FISH, RNA sequencing, luciferase reporter assay, RNA immunoprecipitation, RNA pull-down and rescue experiments, were deployed to detect upstream regulation mechanism of C2CD4A.

Results: C2CD4A was elevated in CRC tissues compared with adjacent normal colorectal tissues. C2CD4A knockdown significantly promoted cell apoptosis and with inhibited proliferation in vitro, and tumorigenicity in vivo, whereas C2CD4A overexpression led to opposite effects. Moreover, circSLC6A6 was upregulated and shown to positively regulate C2CD4A expression via sponging miR-1265. Fundamentally, C2CD4A inhibited p53 signaling pathway through interacting with p53 and increasing its ubiquitination and degradation.
\end{abstract}

Conclusion: Our results identified that circSLC6A6/miR-1265/C2CD4A axis, which was involved in CRC via the p53 signaling pathway, may serve as a therapeutic target for CRC.

Keywords: Colorectal cancer, C2CD4A, p53, circSLC6A6, miR-1265

\section{Background}

Colorectal cancer (CRC), as one of the most common neoplasms, ranks third most frequently diagnosed cancer for males and second for females and is a leading cause of cancer death worldwide [1]. Despite numerous clinical

\footnotetext{
*Correspondence: richard-hc@hotmail.com

†Zeyin Rong, Zai Luo, Zhongmao Fu and Pengshan Zhang contributed equally to this work.

1 Department of General Surgery, Shanghai General Hospital, Shanghai Jiaotong University School of Medicine, 100 Hai Ning Road, Hongkou District, Shanghai 200080, China

Full list of author information is available at the end of the article
}

endeavors for early diagnosis and appropriate treatments of CRC in the past few decades, the prognosis of CRC patients was still less desirable, especially in advancedstage [2]. Hence, further research is urgently required before final goal of working out novel and effective diagnostic methods and identifying precise molecular targets of CRC.

C2CD4A (C2 calcium dependent domain containing $4 \mathrm{~A}$ ) belongs to the $\mathrm{C} 2 \mathrm{CD} 4$ family, which included 3 members, namely C2CD4A, C2CD4B and C2CD4C. Due to its high sequence similarity and coding cells nuclear protein, it was also known as the sequence similarity family original author(s) and the source, provide a link to the Creative Commons licence, and indicate if changes were made. The images or other third party material in this article are included in the article's Creative Commons licence, unless indicated otherwise in a credit line to the material. If material is not included in the article's Creative Commons licence and your intended use is not permitted by statutory regulation or exceeds the permitted use, you will need to obtain permission directly from the copyright holder. To view a copy of this licence, visit http://creativecommons.org/licenses/by/4.0/. The Creative Commons Public Domain Dedication waiver (http://creativeco mmons.org/publicdomain/zero/1.0/) applies to the data made available in this article, unless otherwise stated in a credit line to the data. 
148A (FAM148A) or nuclear localization factor 1 (NLF-l) $[3,4]$. The C2CD4A gene is located on the human chromosome long arm 15q 22.2, which contains 3941 base pairs, 1 intron and 2 exons. Encoded a protein containing a calcium-dependent lazy $\mathrm{C} 2$ domain, $\mathrm{C} 2 \mathrm{CD} 4 \mathrm{~A}$ gene is a nuclear protein with a relative molecular mass of $39 \mathrm{KDa}$. A slice of change in $\mathrm{C} 2$ domain coming along with the variation in C2CD4A gene would affect its encoded protein structure and function. Genome-wide association studies suggested that $\mathrm{C} 2 \mathrm{CD} 4 \mathrm{~A}$ was highly associated with diabetes and was rarely found in tumor cases [5]. Namely, there were research gap in C2CD4A's role in regulating the tumorigenesis of CRC and its mechanism in affecting tumor progression.

P53, a major tumor suppressor gene, was essential for genome integrity and stability [6]. As a vital gatekeeper, p53 was mutated in all sorts of cancers for which it would inhibit cell proliferation, induce cell apoptosis, cell senescence, block cell metastasis and regulate energy metabolism by p53's transcriptional activities [7]. MDM2, a transcriptional target gene of p53, possessed intrinsic E3 ubiquitin ligase activity, was directly bound to p53 and mediated its ubiquitin-dependent proteolysis through its $\mathrm{N}$-terminal, and then formed a negative feedback loop with p53 [8]. Thus, direct or indirect inhibition of the MDM2 activity was crucial for p53 function. Given the critical role of p53 inactivation in the development of CRC, it was significantly important to figure out the molecular mechanism by which p53 was dysregulated in CRC. Our study unveiled that C2CD4A might interact with p53 to increase p53 protein ubiquitin-degradation and thus facilitated the growth of CRC.

Circular RNAs (circRNAs) are defined as an intriguing one in noncoding RNAs (ncRNAs), which are formed by back-splicing or exon skipping and are characterized as covalently closed loop structures with neither $5^{\prime}$ to 3' polarity nor a polyadenylated tail [9]. CircRNAs are insensitive to multiple exonuclease and have displayed a stable state due to its peculiar structure with the potential to be an ideal biomarker for diagnosis of cancer [10]. And it has a particular cell and tissue type or developmental stage-specific expression pattern in eukaryote, and has been proven to take part in the pathology of different diseases [11]. The specificity of the circRNA structure determines its specific biological functions, such as functions in regulation of gene transcription, and protein translation [12]. Relationships between circRNAs and diseases, especially in cancers, have recently been reported, including ones related to gastric cancer, bladder cancer, liver cancer and CRC [13-16]. In this study, we identified an abnormally upregulated circRNA-circSLC6A6 (hsa_circ_0004705) in CRC cells and tissues, which was significantly associated with CRC patients prognosis.
Importantly, circSLC6A6 could bind to miR-1265 and promote the growth of CRC by increasing the expression of $\mathrm{C} 2 \mathrm{CD} 4 \mathrm{~A}$ protein and facilitating the degradation of p53 protein. Therefore, our research was the first uncovered the novel circSLC6A6/miR-1265/C2CD4A/p53 axis in the CRC growth and its molecular mechanism to provide a promising therapeutic targets of CRC.

\section{Materials and methods \\ Patients and tissue samples}

108 paired CRC and adjacent normal tissues were collected from Shanghai General Hospital between 2013 and 2014, and were paraffin embedded for the tissue microarray (TMA) construction (the final TMA contained 106 CRC tissues and 106 adjacent normal tissues). Meanwhile, sixty-three pairs of human CRC fresh tissues and adjacent normal tissues were collected from Shanghai General Hospital after radical surgical resection between 2015 and 2017. After resection, the tissues were transported in liquid nitrogen and stored at $-80^{\circ} \mathrm{C}$ refrigerator for RNA and protein extraction. No patients had received chemotherapy and radiotherapy before surgery. The detailed clinicopathological feature was confirmed by at least two pathologists according to the American Joint Committee on Cance (AJCC). Our research was approved by the Ethics Committee for Clinical Research of Shanghai General Hospital.

\section{Animal experiments}

Male BALB/c athymic nude mice ( 4 weeks old) were randomly divided into 3 groups (five mice per group). Stable transfected RKO cells $\left(5 \times 10^{6}\right)$ were subcutaneously injected into subscapular in each group to establish the CRC xenograft model. Tumor size was measured every week to monitor tumor growth. The tumor volumes were calculated using the formula: $0.5 \times$ length $\times$ width $^{2}$. All mice were sacrificed at 38 days; all tumors were surgically removed and weighed. After fixed in $4 \%$ paraformaldehyde, all tumors were embedded and sliced for IHC staining. All animal experiments were approved by the Institutional Animal Care of Shanghai General Hospital.

\section{Luciferase reporter assay}

Luciferase plasmids (pGL3-Firefly-Renilla containing circSLC6A6 sequence or Mutant sequence, pGL3Firefly-Renilla containing C2CD4A 3'-UTR sequence or Mutant sequence) were purchased from GenePharma (Shanghai, China) and were co-transfected with miR-1265 mimics or inhibitor to indicated cells using Lipofectamine ${ }^{\mathrm{TM}}$ 2000. In short, the transfected cells were lysed with $100 \mu \mathrm{l}$ of passive lysis buffer and the supernatant was collected after centrifuge. Cell samples $(20 \mu \mathrm{l})$ and $100 \mu \mathrm{l}$ of LARII were added into 96-well 
luminometer plates, followed by firefly luciferase activity detection. Then, $100 \mu$ l of Stop \& GloR reagent was added into 96-well luminometer plates to detect Renilla luciferase activity. The ratio of firefly luciferase/Renilla luciferase activity was used to detect the effects of miR1265 on luciferase reporter plasmids. All experiments were independently performed in triplicate.

\section{Fluorescence in situ hybridization (FISH)}

FISH assay was performed to locate circSLC6A6 using a Cy3-labeled probe and to locate miR-1265 using a FAM-labeled probe in HCT8 and RKO cells, respectively. The Cy3-labeled circSLC6A6 probe and FAMlabeled miR-1265 probe were designed and synthesized by GenePharma (Shanghai, China). Cells were seeded in $35-\mathrm{mm}$ glass bottom dishes with $10-\mathrm{mm}$ microwells. After washing with PBS and being fixed with anhydrous ethanol, the cells were treated with $100 \mu \mathrm{l}$ of $0.1 \%$ Triton-100 at room temperature for $15 \mathrm{~min}$. The cells were hybridized with $5 \mu$ l of probe in hybridization buffer (10\% dextran sulfate, $40 \%$ formamide, $4 \times$ saline-sodium citrate (SSC), $1 \times$ Denhardt's solution, $1000 \mathrm{mg} / \mathrm{ml}$ sheared salmon sperm DNA, $10 \mathrm{mM}$ DDT, $1000 \mathrm{mg} / \mathrm{ml}$ yeast transfer RNA) at $37^{\circ} \mathrm{C}$ overnight. Then, cells were continuously washed and dyed with $100 \mu \mathrm{l}$ of DAPI for $20 \mathrm{~min}$. Then, confocal laser scanning microscopy was used to observe the staining. Simultaneously, FISH assay was also performed in TMA, which contained 106 paired CRC samples by Cy3-labeled miR-1265 (Boster, Shanghai). The staining scores were evaluated by two independent pathologists to avoid bias as previously described in our published study [17]. Final, the samples scores were divided into two groups: the high expression group (4-8) and the low expression group (0-3). The sequences of circSLC6A6 and miR-1265 probe for FISH and TMA were listed in Additional file 2: TableS3.

\section{Immunoprecipitation and Mass Spectrometry (IP/MS)}

$293 \mathrm{~T}$ cells transfected with Flag-C2CD4A were lysed in RIPA buffer (20 mM Tris- $\mathrm{HCl}(\mathrm{pH}=8), 137 \mathrm{mM} \mathrm{NaCl}$, 0.5\% Triton X-100, 2 mM EDTA) and protease inhibitor cocktail (Sangon Biotech, Shanghai, China). Cell lysates were incubated with Flag-M2 agarose beads (Sigma) overnight and eluted by Flag peptides (Sigma). The Flag peptide elution was resolved on SDS-PAGE and Coomassie blue stained. Lysates from $293 \mathrm{~T}$ cells transfected with control Flag-vector were served as control. Protein bands specific to the Flag-C2CD4A transfection were digested with trypsin and analyzed by mass spectrometry for protein identification.

\section{Co-immunoprecipitation assay (Co-IP) and ubiquitination assay}

Protein extracts were immunoprecipitated with Protein A+G Agarose beads (Beyotime Biotechnology, Shanghai, China) using appropriate antibodies or control IgG (Sigma, USA). Then, the protein of immunocomplexes were subject to immunoblotting analysis with indicated antibodies. For ubiquitination assay, $293 \mathrm{~T}$ cells were cotransfected with Flag-C2CD4A, HA-P53 and His-ubiquitin. Alternatively, $293 \mathrm{~T}$ cells were co-transfected with sh-C2CD4A, HA-P53 and His-ubiquitin. 24 hour later, $293 \mathrm{~T}$ cells were treated with MG132 $(10 \mu \mathrm{M})$ for 8 hours to inhibit proteasomal degradation. The transfected cells were lysed and centrifuged. Then the supernatants were pulled-down by incubation with Ni-NTA beads, and the protein of immunocomplexes was immunoblotted using anti-HA antibody.

\section{RNA immunoprecipitation (RIP)}

RIP was performed by using Magna RIP ${ }^{\mathrm{TM}}$ RNA-binding protein immunoprecipitation kit (Millipore, Billerica, MA) according to manufacturer's protocol. The Ago2RIP experiments were conducted in RKO cells transiently overexpressing miR-1265 mimics or miR-NC. 48 h later, approximately $1 \times 10^{7} \mathrm{RKO}$ cells were lysed in complete RNA lysis buffer. Then, RKO cell lysates were incubated with RIP immunoprecipitation buffer containing magnetic beads conjugated with human anti- Ago2 or negative control IgG (Millipore, Billerica, MA, USA) at $4^{\circ} \mathrm{C}$ overnight. Each sample was digested with Proteinase $\mathrm{K}$ and then immunoprecipitated RNA were analyzed by qRT-PCR to test the concentration of circSLC6A6. RIP assay was repeated in triplicate.

\section{RNA Pull-down assay}

A pull-down assay was performed as reported [17]. The biotinylated circSLC6A6 probe and miR-1265 probe were designed and synthesized by GenePharma (Shanghai, China) and the sequences were listed in Additional file 2: TableS4. Briefly, cells were collected, lysed, and sonicated. Probe-coated beads were generated by coincubating the circSLC6A6 and miR-1265 probes with probes-M280 streptavidin dynabeads (Invitrogen, USA) at $25^{\circ} \mathrm{C}$ for $2 \mathrm{~h}$. The cell lysates were incubated with circSLC6A6 and miR-1265 probes or oligo probe at $4^{\circ} \mathrm{C}$ overnight. After extensively washed, the RNA complexes bound to the beads were eluted and extracted with the RNAisoPlus (TaKaRa, Japan) and measured by qRT-PCR.

\section{Statistical analysis}

All quantitative data were enumerated by a $x^{2}$ or Fisher's exact test. The associations were analyzed by 
Pearson's test. Comparisons between different groups were analyzed using a paired or unpaired t-test. Survival curves were drawn by Kaplan-Meier method. The SPSS 23.0 software was deployed for statistical analysis. $\mathrm{P}<0.05$ was statistically significant in all tests.

A complete description of the methods, including cell culture and culture conditions, RNA extraction, gDNA extraction, and quantitative real-time polymerase chain reaction (qRT-PCR), uucleic acid electrophoresis and RNase $\mathrm{R}$ treatment, Immunohistochemistry (IHC), cell proliferation assay, apoptosis analysis, western blot analysis, the cancer genome atlas (TCGA) database, GEPIAdataset, Oncomine database, cDNA array analysis, transfection, oligonucleotides and plasmids are available in Additional file 1: Supplementary materials and methods.

\section{Results}

C2CD4A is frequently upregulated in CRC

Through analysis of the CRC clinical samples from the Cancer Genome Atlas (TCGA) database, there were 461 CRC samples with RNAseq and RNAseqV2 data, in which 50 paired samples were with pathological data and RNAseq data (41 paired samples colon adenocarcinoma samples and 9 paired rectum adenocarcinoma samples). Based on the above 50 paired CRC samples, the volcano plots showed CRC related differentially expressed genes were screened (Fig. 1A; Additional file 3: TableS6). Then, we found that $\mathrm{C} 2 \mathrm{CD} 4 \mathrm{~A}$ ( $\mathrm{C} 2$ calcium dependent domai $\mathrm{n}$ containing $4 \mathrm{~A}$ ) expression was significantly higher in CRC tissues than in the paired normal colorectal tissues (19.869 fold change, $P<0.001$ ) (Fig. 1B), and has never been investigated in tumors. GEPIA dataset also comfirmed that $\mathrm{C} 2 \mathrm{CD} 4 \mathrm{~A}$ was higher in CRC tissues than in normal tissues (Fig. 1C). On the basis of Oncomine database (www.oncomine.org), C2CD4A was also significantly higher in 45 colorectal adenocarcinoma and 36 colorectal carcinoma compared with normal tissues (Skrzypczak Colorectal Statistics, 2010), respectively (Fig. 1D). To further confirm the variation of the expression of C2CD4A in CRC tissues, qRT-PCR analysis showed the upregulation of $\mathrm{C} 2 \mathrm{CD} 4 \mathrm{~A}$ in 63 pairs of fresh frozen CRC tissues compared to adjacent noncancer tissues (Fig. 1E). Western blot results further confirmed that $\mathrm{C} 2 \mathrm{CD} 4 \mathrm{~A}$ was upregulated in randomly selected 12 pairs of fresh frozen CRC tissues (Fig. 1F). Meanwhile, C2CD4A mRNA and protein expression were also detected in seven CRC cell lines. C2CD4A was presented with the highest expression in RKO cell and with the lowest expression in HCT8 cell, respectively (Fig. 1G). Hence , we chosen RKO and HCT8 cells for subsequent functional assays.
C2CD4A promoted CRC cell growth in vitro and in vivo

To explore the biological functions of C2CD4A in CRC, knockdown and overexpression vectors (sh-Ctrl, sh1, sh2 and pLVX-Ctrl, pLVX-C2CD4A) were respectively transfected with lentiviral vectors to generate stable knockdown or overexpression cell lines. The effects of knockdown and overexpression on $\mathrm{C} 2 \mathrm{CD} 4 \mathrm{~A}$ were confirmed by qRT-PCR and western blot analysis (Additional file 4: Fig. S1A). Subsequently, CCK-8 assay, EdU assay and clone assay results revealed that the growth rate of RKO cell was noticeably attenuated in the downregulated C2CD4A expression (Additional file 4: Fig. S1B, C and D). Overexpression of C2CD4A led to opposite effects in HCT8 cells (Additional file 4: Fig. S1B, C and D). Next, flow cytometry indicated that downregulated expression of $\mathrm{C} 2 \mathrm{CD} 4 \mathrm{~A}$ increased the percentage of apoptotic $\mathrm{RKO}$ cells, whereas upregulated expression of C2CD4A decreased the percentage of apoptotic HCT8 cells (Additional file 4: Fig. S1E). Based on in vitro findings, we next tested the role of $\mathrm{C} 2 \mathrm{CD} 4 \mathrm{~A}$ in vivo. A xenograft model was constructed by subcutaneous injection of tumor cells in nude mice. The results showed that the tumor volumes were significantly diminished in knockdown groups (Fig. 2A and B). Furthermore, the mean tumor weights of the knockdown groups were significantly lower than those of the control groups (Fig. 2C). We then extracted the total RNA and protein from the tumors in different groups. qRT-PCR and western blot confirmed that the expression of C2CD4A were significantly decreased in knockdown groups (Fig. 2D). Likewise, western blot and IHC staining showed that the levels of Ki-67 were obviously decreased by C2CD4A knockdown, while the levels of Cleaved-caspase3 were markedly elevated (Fig. 2D and E). Taken together, the results above suggested that C2CD4A promoted growth and inbibited apoptosis both in vitro and in vivo in CRC cells.

\section{C2CD4A promotes CRC cells growth via p53 signaling pathway and interacts with p53}

Considering our finding that $\mathrm{C} 2 \mathrm{CD} 4 \mathrm{~A}$ was related to growth, and apoptosis of CRC cells, we aimed to investigate C2CD4A-relevant molecular mechanisms in the process of CRC. We analyzed the correlation between C2CD4A and other genes in CRC using a cDNA array to identify differentially expressed genes (DEGs) between three pairs knockdown and control cells (RKO/shC2CD4A-1 and RKO/sh-Ctrl). According to the results of analysis of DEGs regulated by C2CD4A, 232 and 185 genes were upregulated and downregulated (|fold changes $\mid>1.5, P<0.05$ ), respectively (Fig. 3A), with CDKN1A (p21), BAX, FAS and SESN1 exhibiting the high expression levels and BCL2, Ki67 and TiGAR 


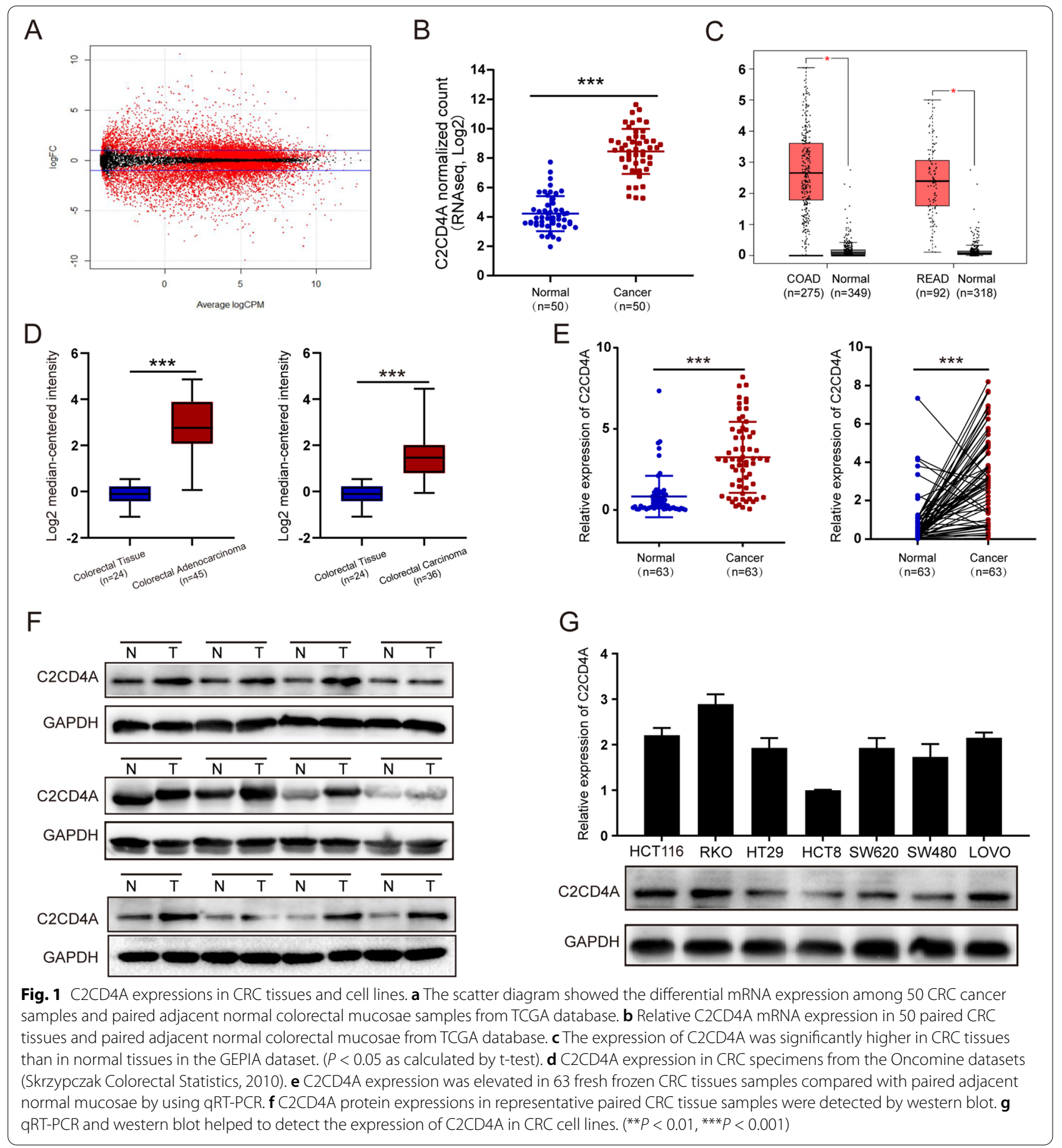

exhibiting the low expression levels (Additional file 2: TableS5). P21, BAX, FAS, SESN1, and TiGAR are all the down-stream genes of p53 transcriptional targets [18, 19]. KEGG pathway analysis of these DEGs revealed that p53 signaling pathway was significantly enriched among the top 10 enrichment-regulated pathways (Fig. 3B). Moreover, we performed the IP/MS to identify key factors associated with C2CD4A. $293 \mathrm{~T}$ cells transfected with Flag-C2CD4A was immunoprecipitated by Flag-M2 agarose beads and the interacting proteins were visualized by Coomassie blue staining after electrophoresis and identified by mass spectrometry (Fig. 3C). We found that one interacting protein turned out to be p53 among the Flag-C2CD4A-interacted proteins associated with cell 


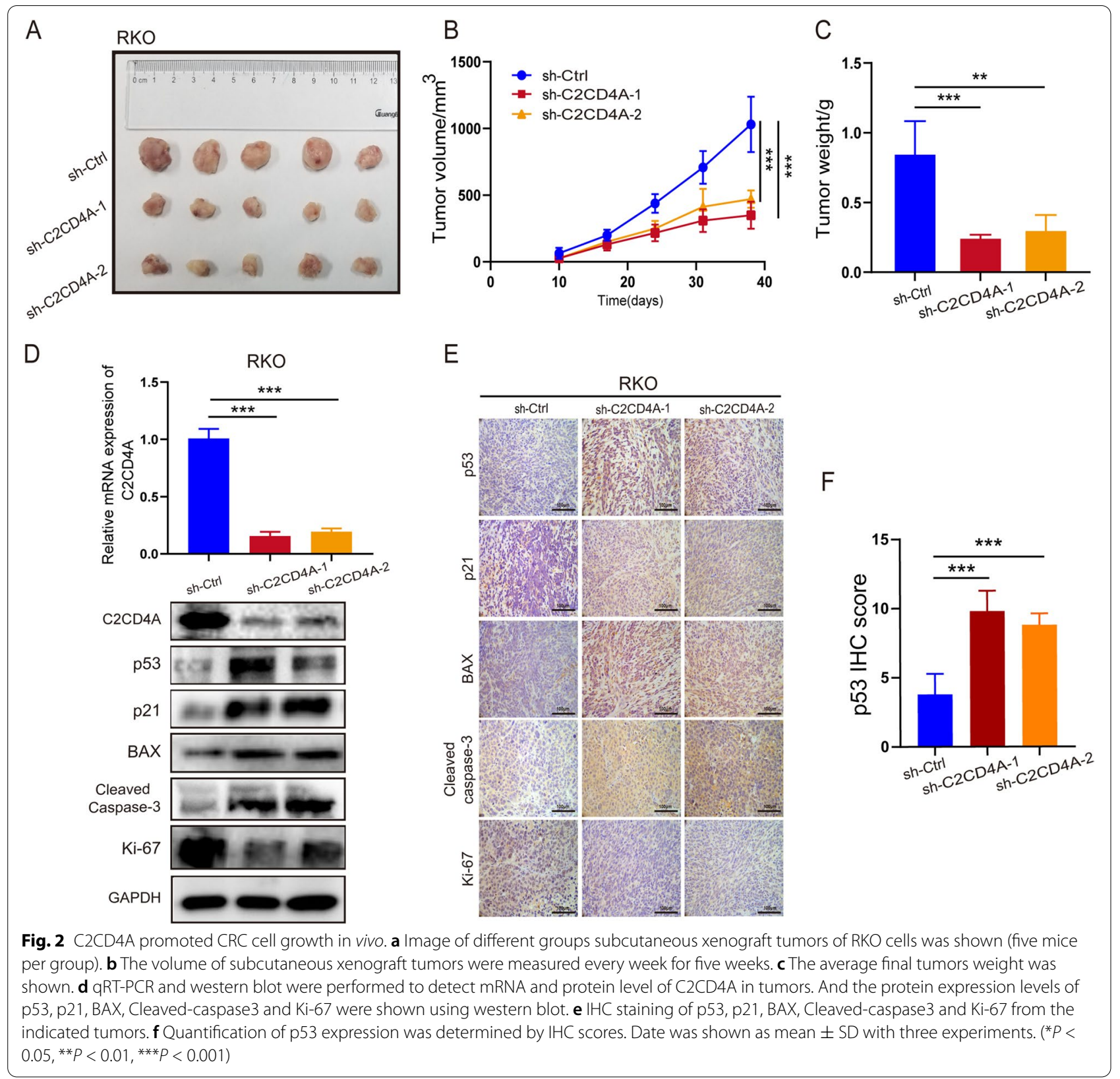

apoptotic (Fig. 3D). As the p53 signaling pathway was of significant influence in the regulation of tumor progression [20], it was inferred that C2CD4A might promote the CRC growth by repressing the $\mathrm{p} 53$ signaling pathway based on our preliminary results. Thus, we further analyzed the correlation between the expression of C2CD4A and p53 down-stream genes. To verify this hypothesis, we measured the expression variation of p53 downstream genes in RKO, HCT116 $\mathrm{p}^{+/+}$and HCT116 p53 ${ }^{-1-}$ cells with stable C2CD4A knockdown. The qRTPCR results showed that C2CD4A knockdown increased p21, BAX, FAS and SESN1 expressions, and decreased TiGAR expression, while the level of p53 mRNA expression remained unchanged in RKO and HCT116 $\mathrm{p}^{+/+}$ cells (Fig. 3E, Additional file 5: Fig. S2A). Likewise, western blot results showed that C2CD4A knockdown had increased the expressions of p53, p21, and BAX level in HCT116 $\mathrm{p}^{+/+}$cells (Fig. 3F). But there is no change in HCT116 $\mathrm{p}^{-1-}$ cells (Fig. 3F). In addition to that, the expression of p53, p21 and Bax were significantly elevated in tumors with $\mathrm{C} 2 \mathrm{CD} 4 \mathrm{~A}$ knockdown in the CRC xenograft mice model using western blot and IHC (Fig. 3D, E). 


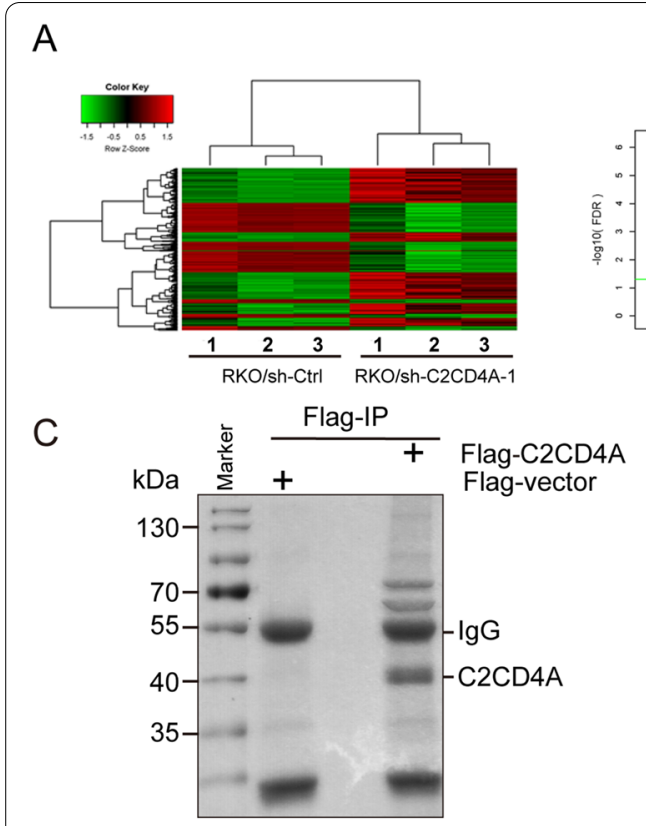

E

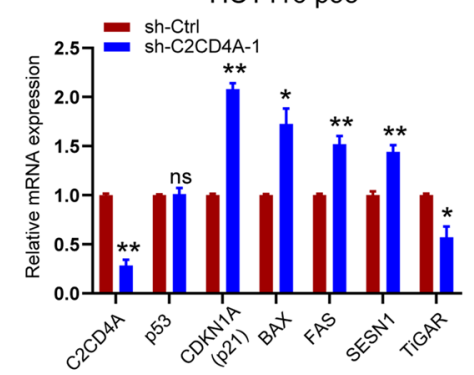

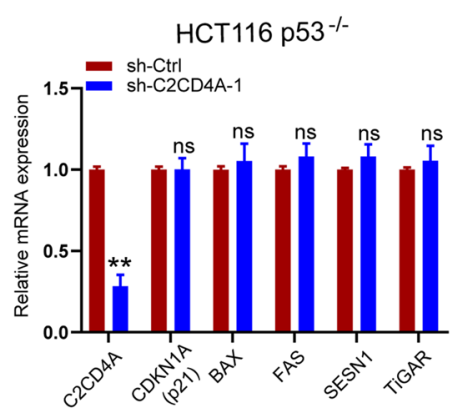

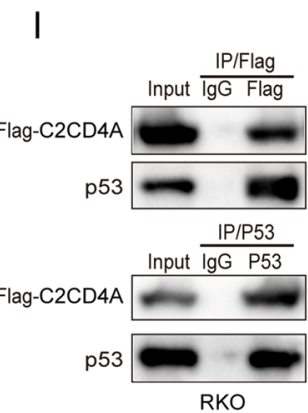

B

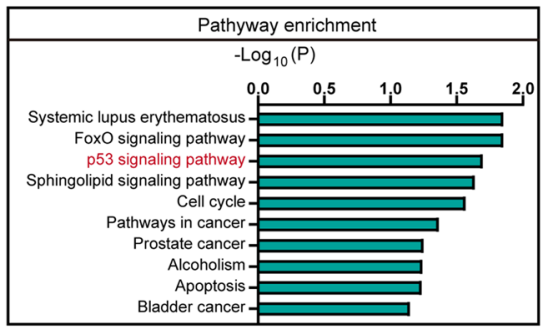

D

\begin{tabular}{ccc}
\hline Protein & \multicolumn{2}{c}{ Quantity of peptides identified } \\
\hline & Flag-C2CD4A & Flag-vector \\
C2CD4A & 31 & 0 \\
P53 & 20 & 0 \\
SLC25A6 & 12 & 0 \\
PPP6C & 4 & 0 \\
AIFM1 & 6 & 0 \\
RPS3 & 13 & 0 \\
HAX1 & 6 & 0 \\
\hline
\end{tabular}

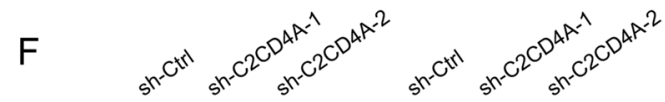

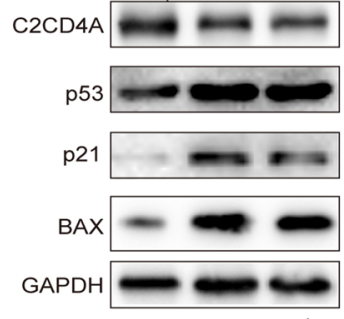

HCT116 $\mathrm{p} 53^{+/+}$

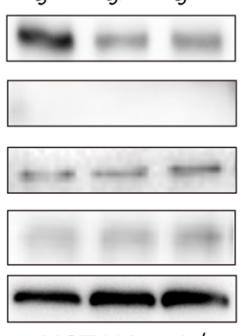

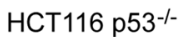

$\mathrm{L}$

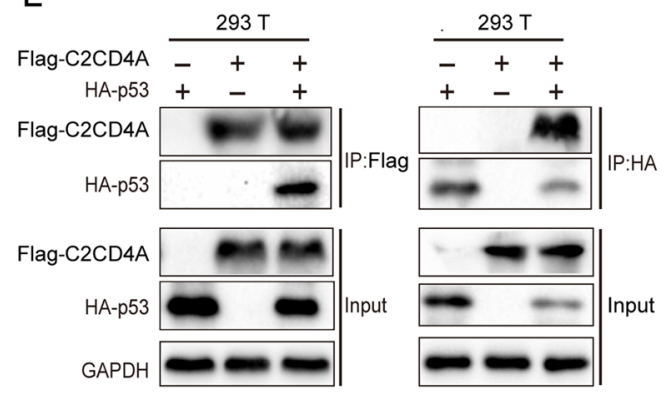

Fig. 3 C2CD4A promoted CRC cells growth via p53 signaling pathway and interacted with p53. a The heatmap and scatter diagram showed the differential expressed mRNA between RKO/sh-C2CD4A (KD-1, 2, 3) and RKO/sh-Ctrl cells (NC-1, 2, 3) screened by human cDNA microarray. $\mathbf{b}$ Top 10 pathways of KEGG analysis based on DEGs. c IP/MS analysis of Flag-C2CD4A-associated proteins. The Flag peptide elution was resolved on SDS-PAGE and Coomassie blue staining. $\mathbf{d}$ Several proteins related to cell apoptosis identified from Flag-C2CD4A IP/MS analysis. e The relative mRNA expressions of C2CD4A, p53, CDKNIA(p21), BAX, FAS, SESN1 and TiGAR were detected using qRT-PCR. f The levels of C2CD4A, p53, CDKNIA(p21) and BAX protein expression were detected using western blot. $\mathbf{g} 293$ T cells were co-transfected with Flag-C2CD4A and HA-P53 at various concentrations, and then Flag and HA expression were detected using western blot. $\mathbf{h ~ H C T 1} 16$ cells were treated with Dox at 1, $2 \mu \mathrm{M}$ for 24 $h$, and treated at $1 \mu \mathrm{M}$ for 12, $24 \mathrm{~h}$. C2CD4A and p53 expressions were detected using western blot. $\mathbf{i}$ The lysates of RKO cells were transfected with Flag-C2CD4A followed by immunoprecipitated using anti-lgG, anti-Flag or anti-p53 antibodies, and immunoprecipitation products were analyzed by western blot with indicated antibodies. I 293 T cells were co-transfected with Flag-C2CD4A and HA-p53. Immunoprecipitation was carried out using anti-Flag or anti-HA antibody, and immunocomplexes were detected using indicated antibodies. (ns showed no significance. ${ }^{*} P<0.05$, ${ }^{* *} P<$ $0.01)$ 
More impotantly, the knockdown of C2CD4A obviously increased the expression of p53 protein (Fig. 3E, F). Collectively, these data suggested C2CD4A might promote the CRC growth and proliferation by repressing the p53 signaling pathway.

We further focused on C2CD4A regulating p53 and its molecular mechanism according to the results of cDNA array and IP/MS. To address the relationship between C2CD4A and p53, $293 \mathrm{~T}$ cells were cotransfected with Flag-C2CD4A and HA-p53 at various concentrations, and then the level of p53 expression was detected. P53 expression decreased with incremental dose of $\mathrm{C} 2 \mathrm{CD} 4 \mathrm{~A}$, showing a dose-dependent manner between C2CD4A and p53 (Fig. 3G). Doxorubicin (DOX) is a DNA damaging agent that has been conformed to increase p53 expression [21]. We used different concentrations of DOX to induce p53 expression, and then observed variations of $\mathrm{C} 2 \mathrm{CD} 4 \mathrm{~A}$ expression in HCT116 cells. The level of p53 expression gradually elevated with the increasing concentration of DOX, whereas the level of C2CD4A expression gradually decreased (Fig. 3H). Therefore, it manifested a significantly concentration-dependent manner between C2CD4A and p53 expression. Above results indicated that $\mathrm{C} 2 \mathrm{CD} 4 \mathrm{~A}$ and $\mathrm{p} 53$ expression were negatively correlated in a dose-dependent manner. Furthermore, we carried out endogenous immunoprecipitation assay to confirm that Flag-C2CD4A interacted with p53 in RKO cells (Fig. 3I). Next, exogenous Flag-C2CD4A and HA-p53 were co-transfected into $293 \mathrm{~T}$ cells, and then Flag-C2CD4A or HA-p53 were respectively immunoprecipitated (Fig. $3 \mathrm{~L}$ ). Taken together, these data demonstrated that interacted with p53.

\section{C2CD4A decreased half-life of $\mathrm{p} 53$ and promoted ubiquitin degradation of $\mathrm{p} 53$ by enhancing the MDM2-p53 interaction}

Former results demonstrated that $\mathrm{C} 2 \mathrm{CD} 4 \mathrm{~A}$ could bind with $\mathrm{p} 53$ and decrease $\mathrm{p} 53$ protein expression. It was speculated that C2CD4A had affected the proteolysis of $\mathrm{p} 53$. HCT116 $\mathrm{p}^{+/+}$cells with stable C2CD4A knockdown were treated with MG132, a specific proteasome inhibitor. Then, the results form western blot analysis indicated that the increased $\mathrm{p} 53$ protein expression caused by C2CD4A knockdown were mainly reversed by MG132 (Fig. 4A). Additionally, $293 \mathrm{~T}$ cells, with stable overexpressed C2CD4A, were also treated with MG132. The results from western blot analysis showed that the decreased expression of p53 caused by C2CD4A overexpression were partially reversed by MG132 (Fig. 4A). Above results indicated that $\mathrm{C} 2 \mathrm{CD} 4 \mathrm{~A}$ medicated the downregulation of $\mathrm{p} 53$ by proteasomal degradation. Then, HCT116 $\mathrm{p} 53^{+/+}$cells with stable $\mathrm{C} 2 \mathrm{CD} 4 \mathrm{~A}$ knockdown were treated with $\mathrm{CHX}$.
The results from time-dependent protein degradation rate assays showed that knockdown of C2CD4A in HCT116 $\mathrm{p} 53^{+/+}$cells significantly decreased the p53 degradation rate, and extended the half-life of endogenous p53 protein (Fig. 4B). Meanwhile, we also successfully overexpressed

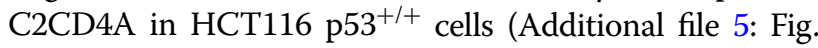
$\mathrm{S} 2 \mathrm{~B}$ ), and $\mathrm{C} 2 \mathrm{CD} 4 \mathrm{~A}$ overexpression shortened the halflife of endogenous p53 protein (Fig. 4C). The results indicated that $\mathrm{C} 2 \mathrm{CD} 4 \mathrm{~A}$ was relevant to stability of $\mathrm{p} 53$ protein. Ubiquitin-proteasome was a highly effective protein degradation pathway in eukaryotic cells [22]. It was widely acknowledged that ubiquitin-mediated degradation through the proteasomal pathway was canonical mechanism for regulating p53 protein homeostasis [23]. Further, it was discussed that whether $\mathrm{C} 2 \mathrm{CD} 4 \mathrm{~A}$ was of influence on p53 ubiquitination. Accordingly, p53 poly-ubiquitination was elevated in $293 \mathrm{~T}$ cells when transfected with FlagC2CD4A (Fig. 4D). Conversely, downregulated C2CD4A expression significantly inhibited p53 poly-ubiquitination (Fig. 4D). E3 ubiquitin ligase murine double minute (MDM2) was not only a transcriptional target of p53 but also the most important negative mediator for p53 protein stability by binding and ubiquitinating $\mathrm{p} 53$ via proteasomal degradation pathway $[24,25]$. To confirm MDM2's role in stimulating C2CD4A's down-regulating p53, p53 protein expression level was monitored after that $293 \mathrm{~T}$ cells were transfected with HA-p53, GFP-MDM2 and Flag$\mathrm{C} 2 \mathrm{CD} 4 \mathrm{~A}$, as determined by immunoprecipitation. Intriguingly, GFP-MDM2 binding to HA-p53 was remarkably elevated in the $293 \mathrm{~T}$ cells with Flag-C2CD4A transfection (Fig. 4D). Further, Flag-C2CD4A coimmunoprecipitated with MDM2 in RKO cells (Additional file 5: Fig. S2C). Concomitantly, Co-IP assay was performed to figure out the interaction among endogenous p53 and GFP-MDM2 in Flag-C2CD4A-overexpressing HCT116 cells. Much more GFP-MDM2 was bound to endogenous p53 when HCT116 cells were transfected with Flag-C2CD4A (Fig. 4E). To further confirm whether $\mathrm{C} 2 \mathrm{CD} 4 \mathrm{~A}$ mediates p53 reduction through MDM2, $293 \mathrm{~T}$ cells, with stable overexpressed C2CD4A, were transfected with siRNAs targeting MDM2 to detect the expression of $\mathrm{p} 53$. The results of western blot showed that $\mathrm{C} 2 \mathrm{CD} 4 \mathrm{~A}$ decreased the expression of $\mathrm{p} 53$, but the expression of $\mathrm{p} 53$ was not remarkably decreased when the silencing of MDM2 (Fig. 4F). Collectively, this data suggested that $\mathrm{C} 2 \mathrm{CD} 4 \mathrm{~A}$ increased p53 poly-ubiquitination and thereby promoted its proteasomal degradation by enhancing the MDM2-p53 interaction

\section{MiR-1265 was downregulated in CRC and suppressed C2CD4A expression by directly binding to the 3'UTR of C2CD4A mRNA}

Accumulating evidences indicated that altered expressions of miRNAs were involved in tumor cell 


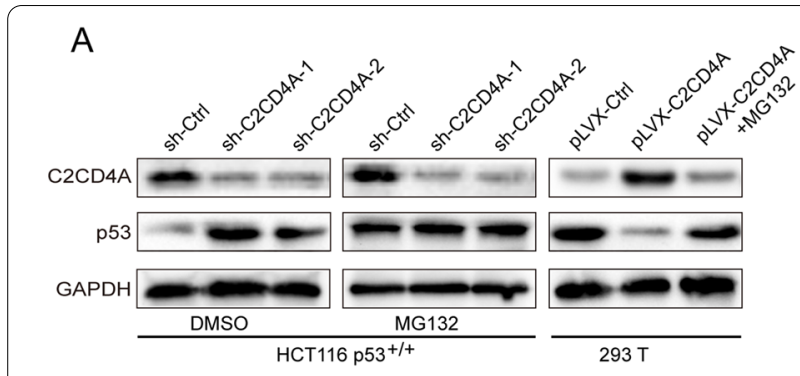

D

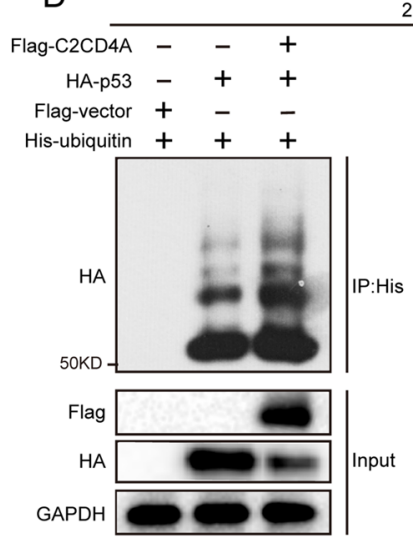

$293 \mathrm{~T}$

$\begin{array}{rllll}\text { sh-C2CD4A-1 } & - & - & + & - \\ \text { sh-C2CD4A-2 } & - & - & - & + \\ \text { HA-p53 } & - & + & + & + \\ \text { Sh-Ctrl } & - & + & - & -\end{array}$

His-ubiquitin ++++

F

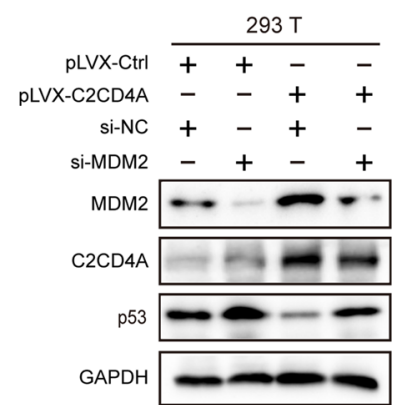

B

\begin{tabular}{lllllll} 
& \multicolumn{6}{c}{ sh-Ctrl } \\
\cline { 2 - 6 } $\mathrm{CHX}(\min )$ & 0 & 20 & 40 & 60 & 90 & 120
\end{tabular}
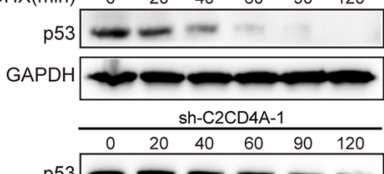

$\mathrm{p} 53$ - -20

GAPDH $=-\infty$

sh-C2CD4A-2
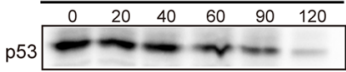

$\mathrm{GAPDH}$

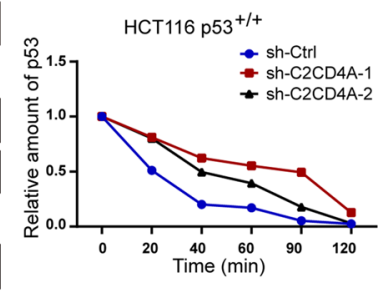

c
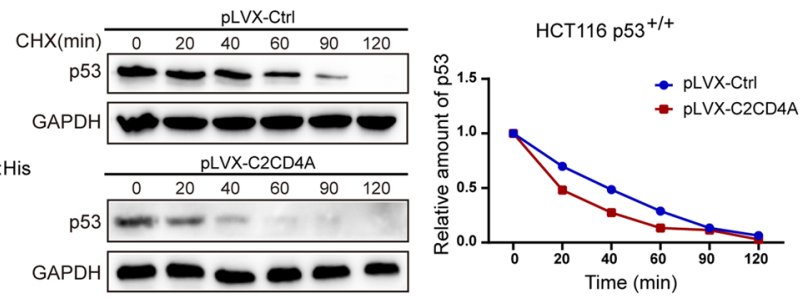

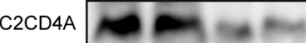

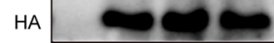

GAPDH

$\mid$

Input

E

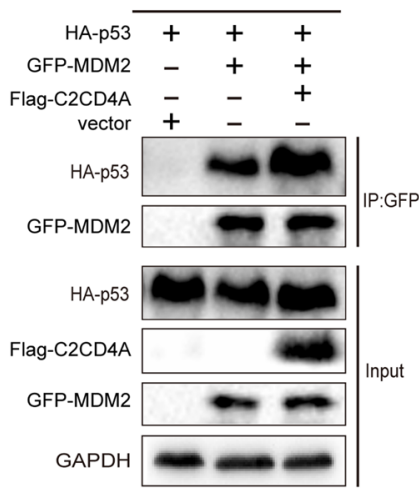

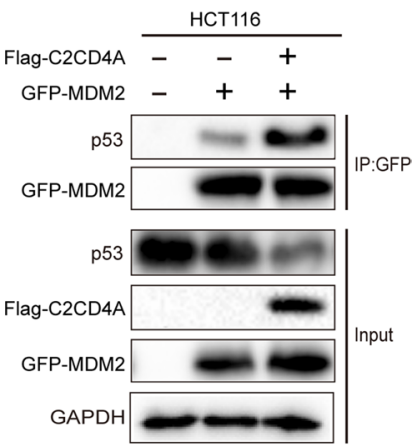

Fig. 4 C2CD4A decreased half-life of p53 and promoted ubiquitin degradation of p53 by enhancing the MDM2-p53 interaction a

C2CD4A-knochdown HCT116 p53+/+ cells were treated with DMSO and MG132. The treatment of DMSO served as a control. 293 T cells, with stable overexpressed C2CD4A, were treated with MG132. The level of C2CD4A and p53 expression were detected using western blot. $\mathbf{b}$, c C2CD4A-knochdown HCT116 p53 $3^{+/+}$cells (b), C2CD4A-overexpression (c) and control HCT116 p53 $3^{+/+}$cells were transfected with CHX and harvested at indicated points after CHX treatment, respectively. The level of C2CD4A and p53 expression were detected using western blot. $\mathbf{d}$ 293 T cells were co-transfected with Flag-C2CD4A, HA-p53, His-ubiquitin or vector. His was immunoprecipitated using anti-His antibody, and p53 ubiquitination was detected using anti-HA antibody (left). 293 T cells were co-transfected with His-ubiquitin, sh-C2CD4A-1, sh-C2CD4A-2, HA-p53 or vector. His was immunoprecipitated using anti-His antibody, and P53 ubiquitination was detected using anti-HA antibody (right). co-immunoprecipitation revealed the polyubiquitination of HA-p53. Co-IP assays revealed the polyubiquitination of HA-p53. e 293 T and HCT1 16 cells were co-transfected with the indicated plasmids, and Co-IP assays were performed using anti-GFP antibody to detect the interaction between p53 and MDM2. f The p53, MDM2, C2CD4A expressions were detected in the tranfected 293 T cells using western blot

proliferation, apoptosis, metabolism, and metastasis [26]. Based on our previous studies on miRNAs in gastrointestinal tumors $[13,17]$, it was aimed to further figure out whether the overexpression of C2CD4A in CRC was caused by a specific miRNAs dysregulation. Using bioinformatics prediction tools, such as
Targetscan, miRWalk and Microrna.org, among those miRNAs which could bind with 3'UTR of C2CD4A, we preliminarily screened out hsa-miR-339-3p, hsa-miR-3153, hsa-miR-361-5p, hsa-miR-3186-5p, hsa-miR-764, hsa-miR-556-3p, hsa-miR-1265, hsamiR-4290 and hsa-miR-3159 as potential regulators 


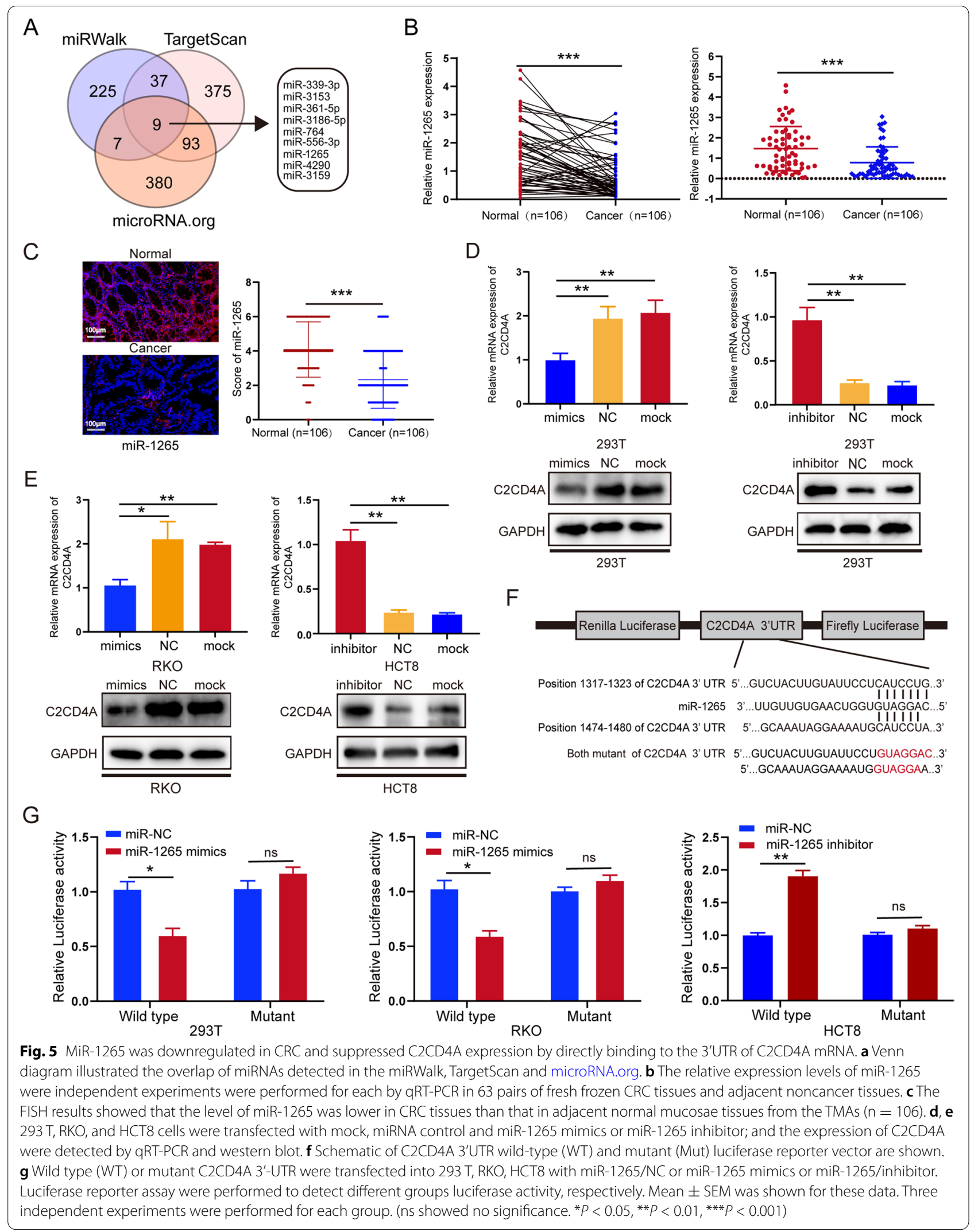


of C2CD4A (Fig. 5A). For above potential regulators, it was found that hsa-miR-339-3p, hsa-miR-361-5p and miR-1265 had been reported in different tumors [27-30], in which hsa-miR-339-3p and hsa-miR361-5p were lowly expressed in CRC and had assumed the role of tumor suppressing [31, 32]. Next, we used qRT-PCR to detect the expression of miR-339-3p, hsa-miR-361-5p and miR-1265 in 63 pairs fresh frozen CRC tissues, and found that miR-1265 was significant lower in 63 pairs fresh frozen CRC tissues (48/63, 76.2\%) compared with those adjacent noncancer tissues (Fig. 5B, Additional file 6: Fig. S3A). Next, qRT-PCR results indicated that miR-1265 expression was negatively correlated with C2CD4A mRNA expression in the above CRC samples (Additional file 6: Fig. S3B). However, C2CD4A expression was not significantly correlated with miR-339-3p and miR-361-5p expression (Additional file 6: Fig. S3B). Hence it was speculated that miR-1265 might be the upstream miRNA that regulate $\mathrm{C} 2 \mathrm{CD} 4 \mathrm{~A}$ expression. Previous studies confirmed that miR-1265 was considered as a tumor suppressor in bladder cancer, glioma, and osteosarcoma [27, 28, 33]. Notably, the results of FISH assay using TMA showed that the expression of miR-1265 was higher in adjacent noncancer tissues than in paired CRC tissues (Fig. 5C). However, there was no obvious correlations between miR-1265 expression and different clinicopathological features (Additional file 7: Table S7), including the prognosis of CRC patients (data not shown). To explore whether miR-1265 inhibited the expression of C2CD4A, qRT$\mathrm{PCR}$ and western blot analysis indicated that C2CD4A mRNA and protein expression was significantly downregulated in the miR-1265/mimics (Fig. 5D, E). However, miR-1265/inhibitor induced the opposite effects (Fig. 5D, E). Then, the 3'UTR of C2CD4A, which was predicted to interact with miR-1265, was cloned into a luciferase reporter vector (wild type, WT). Additionally, a reporter carrying two mutated miR-1265 binding sites was also created (Mutant) (Fig. 5F). Luciferase results indicated that inhibited luciferase activity in the cell lysates had transfected with miR-1265/mimics comparing to those transfected with the negative control mimics in the $293 \mathrm{~T}$ and RKO cells, and were of no effect on the RKO cells with Mutant vectors (Fig. 5G). However, the luciferase activity in the cell lysates was increased when transfected with miR-1265/inhibitor in the HCT8 cells, but did not strengthen luciferase activity in those with Mutant vectors (Fig. 5G). Then, ectopic expression of miR-1265 reduced the levels of p53 and Phosphorylation of p53 (Ser15) protein, and the opposite effects were observed when downregulation of miR-1265 (Additional file 9: Fig. S5E). The aforementioned consequences indicated that miR-1265 targeted directly to the 3'UTR of C2CD4A mRNA and suppressed C2CD4A mRNA translation in CRC.

\section{MiR-1265 inhibits cell growth and promotes apoptosis in vitro}

To explore the possible underlying biological mechanism of miR-1265 function in CRC cells, the results from cell counting assays which included CCK-8 assays, clone formation assays and EdU assays demonstrated that miR-1265/mimics significantly inhibited the growth and proliferation of RKO cells (Additional file 8: Fig. S4A, B and C), while miR-1265/inhibitor promoted growth and proliferation of HCT8 cells (Additional file 8: Fig. S4A, B and C). Meanwhile, miR-1265/mimics increased the proportion of apoptosis cells in RKO (Additional file 8: Fig. S4D), while miR-1265/inhibitor decreased apoptosis cells in HCT8 (Additional file 8: Fig. S4D). In conclusion, miR-1265 had inhibited cell growth and promoted cell apoptosis in CRC.

\section{CircSLC6A6 is overexpressed in CRC}

Accumulating studies confirmed that circRNAs was of great significance in tumor progression [34]. To identify the underlying dysregulated circRNAs in the CRC, RNA-seq analyses was performed to detect differentially expressed circRNAs between in 12 paired fresh frozen CRC tissues and corresponding adjacent normal colorectal mucosae tissues. We found that 373 differentially expressed circRNAs were identified with fold changes $>2$ or $<0.5(P<0.05)$ (Fig. 6A). Differentially expressed circRNAs which included hsa circ_0023984, hsa_circ_0008192, hsa_circ_0008694, hsa_circ_0004705 and hsa_circ_0001153 were highly expressed in CRC. Combined with CircInteractome database (https://circinteractome.nia.nih.gov/), bioinformatics analysis revealed that hsa_circ_0004705, termed as circSLC6A6, had possessed miR-1265 binding sites (Fig. 7D). Therefore, we focused on circSLC6A6, which was spliced from the SLC6A6 gene on chr3: 3p21: 45,695,388-45,699,581, with an ultimate length of 610 nucleotides. By comparing SLC6A6 mRNA sequences with the expected sequences of circSLC6A6 acquired from circBase (http://www.circb ase.org/), schematic diagram illustrated that circSLC6A6 was originated from exons 3, 4 and 5 of its parental SLC6A6 pre-mRNA (Fig. 6B). It was further confirmed the splicing sites via Sanger sequencing 


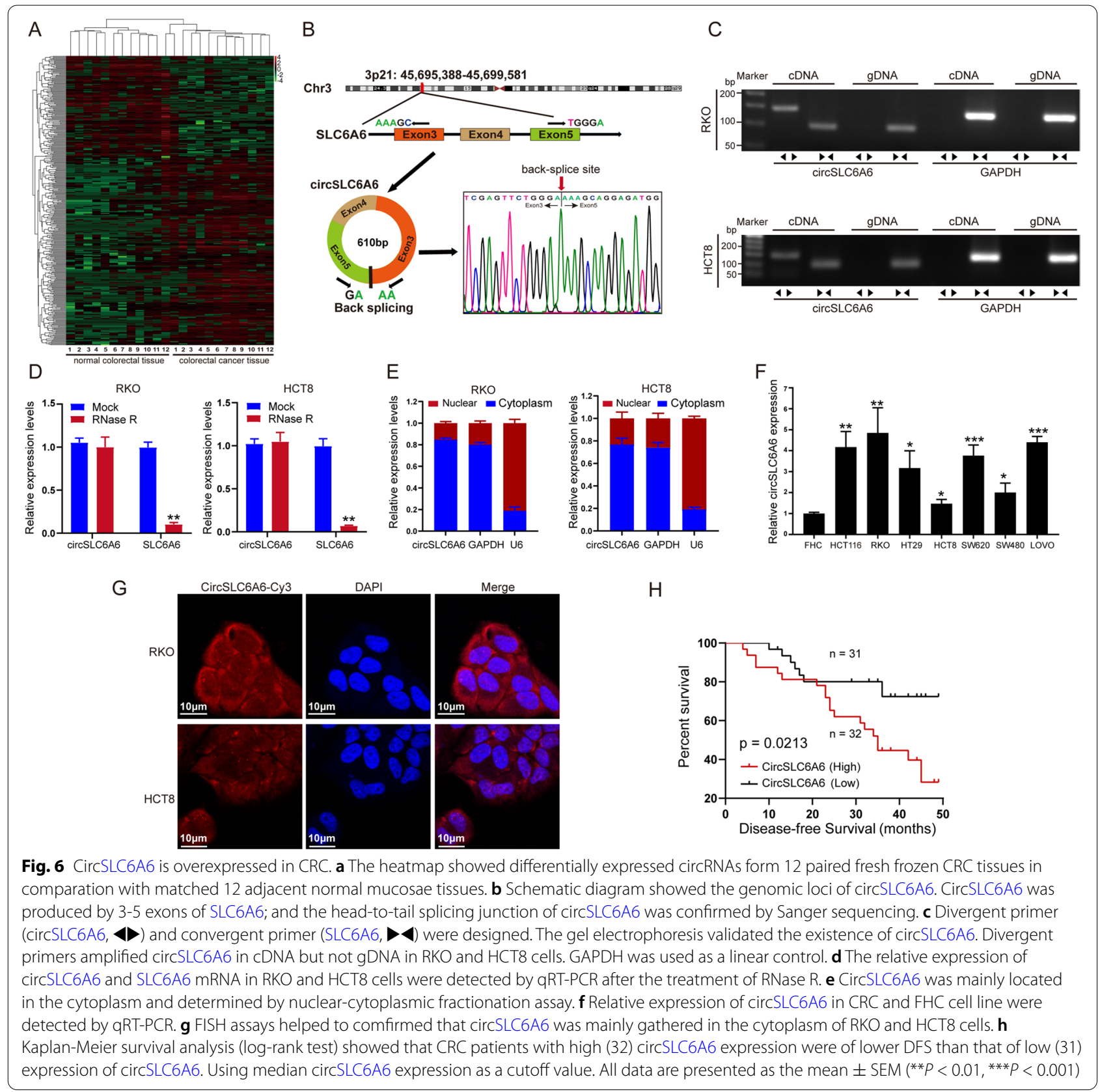

(Fig. 6B). However, head-to-tail splicing could be the result of not only trans-splicing but also genomic rearrangements [35]. To mark off above two varieties, special divergent primers to amplify circSLC6A6 and convergent primers for circSLC6A6 mRNA were designed. cDNA and gDNA were extracted separately from RKO and HCT8 cells which were subjected to nucleic acid electrophoresis detection. It was indicated that hat circSLC6A6 could be detected only in cDNA (141-bp fragment), with no products were detected in the extracted gDNA (Fig. 6C). To verified the stability of circSLC6A6, total RNA treatment of RNase R. qRT-PCR results showed that RNase $\mathrm{R}$ failed to digest circSLC6A6, further confirming the circular structure of circSLC6A6 (Fig. 6D). Furthermore, the results of nuclear cytoplasmic fractionation illustrated that circSLC6A6 was mainly positioned in the cytoplasm (Fig. 6E). Next, circSLC6A6 was prominently expressed in CRC cell lines in comparation with FHC cell line via qRT-PCR. Among CRC cell lines, RKO showed the 


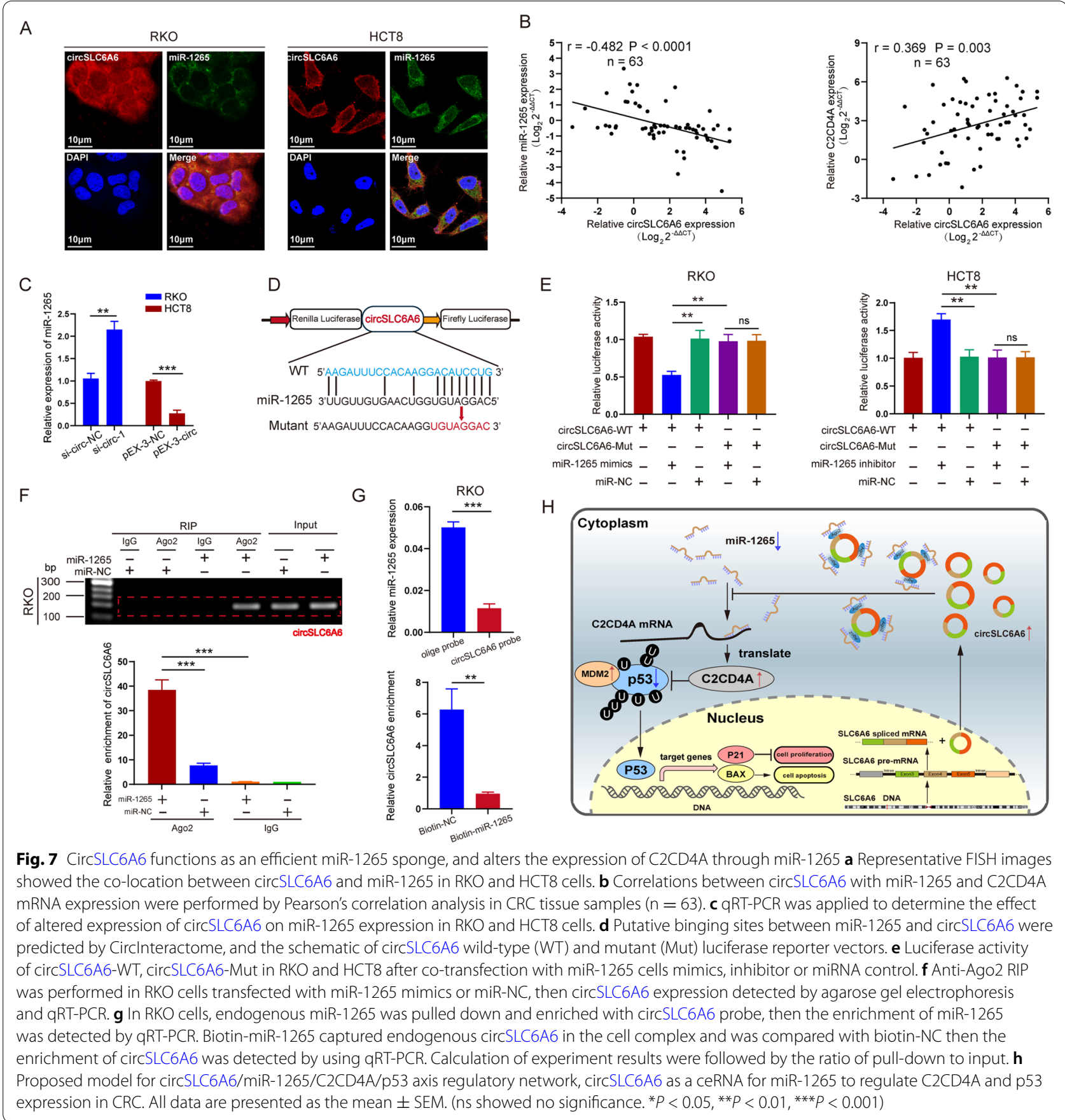

highest level of circSLC6A6, whereas HCT8 the lowest level (Fig. 6F). Additionally, the FISH results also showed that circSLC6A6 was located predominantly in the cytoplasm (Fig. 6G). All these results confirmed that circSLC6A6 was highly stable in cytoplasm of CRC cells. CircSLC6A6 was markedly upregulated in CRC tissues compared with adjacent non-tumor tissues (Additional file 9: Fig. S5A, B). More importantly, the patients with higher circSLC6A6 expression harbor a relatively worse DFS (Fig. 6I). All those findings suggested that circSLC6A6 was upregulated both in CRC cells and tissues and was a vital role in diagnosis and prognosis of CRC. 


\section{CircSLC6A6 functions as an efficient miR-1265 sponge, and alters the expression of C2CD4A and p53 through miR-1265}

Numerous studies have reported that circRNAs might function as miRNA sponges to abrogate the functions of miRNAs $[35,36]$. Hence, we explored whether circSLC6A6 promoted CRC growth and proliferation by sponging miRNAs. Firstly, by utilizing CircInteractome database, it was predicted that the potential target miRNAs of circSLC6A6 and the possible downstream miRNAs. It was indicted that miR-1265 shared binding site with circSLC6A6 attracted our attention. The FISH results revealed that co-location of circSLC6A6 and miR-1265 were mainly located in cytoplasm (Fig. 7A). In addition, the results from qRT-PCR indicated that circSLC6A6 expression was negatively correlated with miR1265 expression, and positively associated with C2CD4A expression in 63 pairs CRC tissues (Fig. 7B), which indicated that circSLC6A6 might be involved in regulating miR-1265 and C2CD4A expression. All the results revealed that circSLC6A6 might interact with miR-1265 in CRC. To address the regulation of circSLC6A6 on miR-1265, three circSLC6A6-targeting siRNAs (si-circ-1, si-circ-2, si-circ-3) and a circSLC6A6 overexpression vector (pEX3-circ) were constructed to alter the expression of circSLC6A6. The result of qRT-PCR showed that the altered expression of circSLC6A6 had no significantly effect on the expression of SLC6A6 mRNA (Additional file 9: Fig. $54 \mathrm{C}, \mathrm{D})$. Then, we monitored the effect of altered circSLC6A6 expression on miR-1265 expression. Downregulation of circSLC6A6 could decrease miR-1265 expression and upregulation one could increase miR1265 expression in RKO and HCT8 cells, respectively (Fig. 7C). Additionally, full length circSLC6A6 sequences (WT, wild type) and sequences with mutant binding site (Mutant) were constructed (Fig. 7D). Luciferase reporter assay results indicated that miR-1265/inhibitor relatively strengthened the luciferase activity of vector containing wild circSLC6A6 but did not alter the luciferase activity of the vector containing mutant circSLC6A6 (Fig. 7E). Conversely, miR-1265/mimics relatively subdued the luciferase activity of vector with wild circSLC6A6 but did not decrease the vector with mutant circSLC6A6 (Fig. 7E).

Next, RIP assays using anti-Ago2 or IgG were carried out in RKO cells with transiently transfected with miR-1265 mimics or controls, followed by agarose gel electrophoresis qRT-PCR and analysis for circSLC6A6 levels (Fig. 7F). The RIP results showed that circSLC6A6 pulled down with anti-Ago2 antibody was significantly enriched in RKO cells after being transfected with miR1265 mimics compared to controls, suggesting that miR-1265 could directly target circSLC6A6 in AGO2 manner. Moerover, RNA pull-down assay indicated that endogenous miR-1265 or circSLC6A6 were significantly pulled down by biotinylated probes against miR-1265 or circSLC6A6, displaying the existence of circSLC6A6/ miR-1265 complexes. The results indicated that specific overexpression miR-1265 was detected in the circSLC6A6 pull-down pellet. Moreover, more circSLC6A6 was captured in the biotin-miR-1265 groups than in the biotin-negative control (NC) groups (Fig. 7G), suggesting that circSLC6A6 served as an effient miR-1265 sponge. Moreover, downregulation of circSLC6A6 markedly attenuated the expression of $\mathrm{C} 2 \mathrm{CD} 4 \mathrm{~A}$ and MDM2, and increased the expression of p53, p21, Bax, and MDM2 (Additional file 9: Fig. S5F). Conversely, upregulation of circSLC6A6 enhanced the expression of C2CD4A and MDM2, and decreased the expression of p53, p21, Bax, and MDM2, which indicated that circSLC6A6 positively regulated the expression of $\mathrm{C} 2 \mathrm{CD} 4 \mathrm{~A}$ (Additional file 9: Fig. S5G) . However, miR-1265/inhibitor partly reversed the decreased expression $\mathrm{C} 2 \mathrm{CD} 4 \mathrm{~A}$ induced by downregulated circSLC6A6 expression, and $\mathrm{miR}-1265 / \mathrm{mim}$ ics reversed the overexpression of $\mathrm{C} 2 \mathrm{CD} 4 \mathrm{~A}$ induced by upregulated circSLC6A6 expression, indicating that miR-1265 could reverse the regulation of circSLC6A6 on C2CD4A expression (Additional file 9: Fig. S5 F, G). Therefore, we demonstrated that circSLC6A6 acted as miR-1265 sponge and lessen the repressive effect of miR1265 on C2CD4A and P53 expression, thereby upregulating $\mathrm{C} 2 \mathrm{CD} 4 \mathrm{~A}$ and downregulating P53 expression. Collectively, these results unveiled a new mechanism that circSLC6A6 bind directly to miR-1265 and promote the proliferation of $\mathrm{CRC}$ through enhancing $\mathrm{C} 2 \mathrm{CD} 4 \mathrm{~A}$ expression.

\section{Circ SLC6A6 promotes cell growth and inhibits apoptosis in vitro}

To investigate the biological functions of circSLC6A6 in CRC cells, results from cell proliferation assays, including CCK-8 assays, EdU assays and clone formation assays, demonstrated that downregulated circSLC6A6 significantly suppressed the growth and proliferation of RKO cells (Additional file 10: Fig. S6A, B and C), while the enforced expression of circSLC6A6 remarkably promoted the growth and proliferation of HCT8 cells (Additional file 10: Fig. S6A, B and C). Meanwhile, downregulated circSLC6A6 increased the rate of apoptosis cells in RKO (Additional file 10: Fig. S6D), while enforced expression of circSLC6A6 decreased the rate of HCT8 apoptosis cells (Additional file 10: Fig. S6D). Collectively, these findings suggested that circSLC6A6 promoted cell growth,and inhibited cell apoptosis in CRC. 


\section{Discussion}

In our present results, C2CD4A was significantly upregulated in CRC tissues and cells. To elucidate the mechanism of $\mathrm{C} 2 \mathrm{CD} 4 \mathrm{~A}$ in resulting in CRC growth, we observed that $\mathrm{C} 2 \mathrm{CD} 4 \mathrm{~A}$ integrated with p53 to facilitate ubiquitin degradation of p53 through increasing interaction of MDM2 with p53. Additionally, our results showed that circSLC6A6 regulated $\mathrm{C} 2 \mathrm{CD} 4 \mathrm{~A}$ and $\mathrm{p} 53$ expression by sponging miR-1265 to promote growth of CRC cells. Our findings was the first to explore the function of circSLC6A6/miR-1265/C2CD4A/p53 axis in the growth of CRC.

C2CD4A, which usually conferring human diabetes susceptibility was firstly reported as a gene relevant to CRC in our study related to CRC. It was found that the knockdown or overexpression of C2CD4A was capable of inhibiting or facilitating the growth of CRC. To further reveal the roles of $\mathrm{C} 2 \mathrm{CD} 4 \mathrm{~A}$ in $\mathrm{CRC}$, our attention was focused on the downstream molecular mechanisms underlying $\mathrm{C} 2 \mathrm{CD} 4 \mathrm{~A}$. The expression of $\mathrm{C} 2 \mathrm{CD} 4 \mathrm{~A}$ in RKO cells was knocked-down, and cDNA arrays were performed to screen for altered genes. According to the results of analysis of DEGs by KEGG analysis, we speculated that $\mathrm{C} 2 \mathrm{CD} 4 \mathrm{~A}$ might promote the CRC growth and proliferation by repressing the p53 signaling pathway. IP/MS also confirmed that C2CD4A may interact with p53. It was being well acknowledged that ubiquitination-induced proteasomal degradation of p53 was essential to maintain p53 protein homeostasis, whose disruption was a molecular marker for cancer in recent 40 years $[37,38]$. It was demonstrated by Wang et al. that TRIM67, a transcriptional target of $\mathrm{p} 53$, functioned as a tumor suppressor by directly binding with the $\mathrm{C}$ terminus of $\mathrm{p} 53$ and protecting it from MDM2-mediated ubiquitination in CRC [39]. Yet, the regulation aberrant expression of $\mathrm{p} 53$ protein had not been thoroughly studied [40]. In this study, we firstly described the aberrant upregulated C2CD4A in CRC tissue and its critical role in regulating p53 protein stability, C2CD4A regulated the expression of p53 target genes, including $\mathrm{p} 21$ and BAX. We also found that C2CD4A shortened half-life of $\mathrm{p} 53$, suggesting that C2CD4A might promote CRC oncogenesis through decreasing p53 function. Furthermore, p53 accumulation was enhanced in C2CD4A-silenced HCT116 p53 $3^{+/+}$cells. Next, we confirmed that C2CD4A, as a novel regulator of the p53 pathway, interacted with p53 and promoted p53 ubiquitination Thus, C2CD4Amediated 553 degradation implied that C2CD4A might be critical for CRC growth and tumorigenesis. The E3 ubiquitin ligase MDM2 had acted as a vital negative regulator of p53 protein level and activity among plenty of proteins which involved in p53 regulation
[24]. MDM2 was bound to p53 and ubiquitinates it proteasomal degradation. Intriguingly, the upregulated C2CD4A expression was able to increase the binding of MDM2 and p53 to facilitate p53 degradation. These results indicated that $\mathrm{C} 2 \mathrm{CD} 4 \mathrm{~A}$ mediated $\mathrm{p} 53$ ubiquitin-degradation through increasing the interaction of MDM2 with p53. MDM2 may be a vital factor in C2CD4A mediated-p53 ubiquitination.

Numerous studies confirmed that aberrantly expressed miRNAs had regulated CRC development and progression [41, 42]. The biological function of miR-1265 widely considered as a tumor-suppressive role in several cancers and its molecular targets in the process of tumorigenesis had been illustrated in several studies $[27,28,33]$. In present study, we found that miR1265 was significantly downregulated in CRC tissues. Subsequently, our study demonstrated that miR-1265 was directly bound to 3'UTR of C2CD4A to decrease C2CD4A expression and therefore inhibited the growth of CRC. Luciferase reporter assay also verified this direct interaction between miR-1265 and C2CD4A in CRC cells. Currently, circRNAs served as the most popular topic in the field of carcinogenesis and cancer progression [43]. Accumulating evidence disclosed that circRNAs were involved in the regulation of cancer cell proliferation, survival, migration and differentiation $[13,16,44,45]$. Up till now, the miRNA sponge was still the most common mechanism for circRNA to exert its biological functions $[13,46]$. Particularly, our previous study indicated that circMLLT10 served as a sponge of miR-509-3-5p, and promoted gastric cancer cell progression through increasing the expression of GINS4 and then activating Rac1 and CDC42 [44]. Moreover, our recently study demonstrated that circCCDC9 directly sponged miR-6792-3p to relieve the repressive effect of miR-6792-3p on its target CAV1, and then to suppress the progression of gastric cancer [17]. CircITCH up-regulates p21 and PTEN through sponging miR-17 and miR-224, which suppressed the aggressive biological behaviors of bladder cancer [47]. In this study, it was identified that circSLC6A6 derived from SLC6A6 gene, which was highly expressed in CRC tissue and was capable of sponging miR-1265, and thus elevated C2CD4A expression and stimulated CRC growth and proliferation. There were several pieces of evidence implicating that circSLC6A6 functioned as a sponge of miR-1265 to regulate C2CD4A in CRC. First, circSLC6A6 expression negatively correlated with miR-1265, whereas positively correlated with $\mathrm{C} 2 \mathrm{CD} 4 \mathrm{~A}$ expression in 63 fresh frozen CRC tissues. Second, downregulated circSLC6A6 would separately lead to a higher miR1265 and lower C2CD4A expression and up-regulated circSLC6A6 resulted in opposite effects. Third, 
bioinformatics analyses and luciferase reporter assays verified this prediction. Fourth, miR-1265 inhibition reversed the effect of downregulated C2CD4A expression caused by knockdown of circSLC6A6, and overexpression of miR-1265 reversed the effect of upregulated C2CD4A expression caused by overexpression of circSLC6A6. Finally, circSLC6A6 could regulate C2CD4A expression, thus leading to the C2CD4A-reduced p53 degradation and inhibiting downstream pathway (Fig. 7H). In conclusion, circSLC6A6 was demonstrated to be an important candidate oncogene in CRC and was involved in the prognosis of CRC patients. CircSLC6A6 might act as a miR-1265 sponge to abolish the inhibitory effects on C2CD4A expression, which eventually promoted the growth of CRC cells. Our study revealed that the circSLC6A6/miR-1265/C2CD4A/p53 axis was involved in the pathogenesis and development of CRC, indicating that this axis might be a novel therapeutic target in patients with CRC.

\section{Conclusion}

To conclude, for the first time, our results reveals that circSLC6A6/miR-1265/C2CD4A axis, which was involved in CRC growth via the p53 signaling pathway, may provide new ideas and targets for CRC treatment.

\begin{abstract}
Abbreviations
AJCC: American Joint Commission on Cancer; CHX: cycloheximide; CircRNA: Circular RNA; C2CD4A: C2 calcium dependent domain containing 4A; ceRNA: Competing endogenous RNA; CRC: Colorectal cancer; DEGs: differentially expressed genes; DFS: Disease-free survival; DOX: Doxorubicin; FISH: Fluorescence in situ hybridization; IHC: Immunohistochemistry; IP/MS: Immunoprecipitation and Mass Spectrometry; miRNA: MicroRNA; KEGG: Kyoto Encyclopedia of Genes and Genomes; qRT-PCR: Real-time quantitative polymerase chain reaction; OS: Overall survival; RIP: RNA immunoprecipitation; TMA: Tissue microarray; 3'UTR: 3'-untranslated region; SIX1: Sine oculis homeobox homolog 1; SLC6A6: Solute carrier family 6 member 6; TRIM67: Tripartite motif containing 67.
\end{abstract}

\section{Supplementary Information}

The online version contains supplementary material available at https://doi. org/10.1186/s13046-021-02126-y.

\footnotetext{
Additional file 1.

Additional file 2.

Additional file 3.

Additional file 4.

Additional file 5 .

Additional file 6.

Additional file 7.

Additional file 8.

Additional file 9.

Additional file 10 .
}

Acknowledgements

Not applicable.

\section{Authors' contributions}

C. H. and ZY. R. designed the experiments and revised the manuscript. ZY. R. performed the main experiments and wrote the manuscript. Z. L., ZM. F., PS. Z., gathered and analyzed the raw data. JM. Z., TF. L., ZL. Z., ZL.Y. Q.L.and ZJ. Q. performed the experiments. All authors provided supervision and approved the manuscript.

\section{Funding}

This work was supported by grants from the National Natural Science Foundation of China (81772526 and 82072662 ) received by C. H., Shanghai Municipal Education Commission-Gaofeng Clinical Medicine Grant Support (20161425) received by C. H., Shanghai Jiaotong University Medical Cross Fund (YG2017MS28) received by C. H., Shanghai.

\section{Availability of data and materials}

The data sets used in this study can be obtained from the corresponding author on reasonable request.

\section{Declarations}

\section{Ethics approval and consent to participate}

This work was ratified by the Ethics Committee of Shanghai General Hospital. All animals were used according to the Shanghai General Hospital Animal Care and Use Guidelines. Written informed consents were obtained from all patients.

\section{Consent for publication}

All authors consent for publication.

\section{Competing interests}

The authors declare no conflicts of interest in this article.

\section{Author details}

'Department of General Surgery, Shanghai General Hospital, Shanghai Jiaotong University School of Medicine, 100 Hai Ning Road, Hongkou District, Shanghai 200080, China. ${ }^{2}$ Department of Medical Oncology, Shuguang Hospital, Shanghai University of Traditional Chinese Medicine, Shanghai, China.

${ }^{3}$ Academy of Integrative Medicine, Shanghai University of Traditional Chinese Medicine, Shanghai, China.

Received: 16 June 2021 Accepted: 4 October 2021

Published online: 16 October 2021

References

1. Sung H, Ferlay J, Siegel RL, Laversanne M, Soerjomataram I, Jemal A, et al. Global Cancer Statistics 2020: GLOBOCAN Estimates of Incidence and Mortality Worldwide for 36 Cancers in 185 Countries. CA Cancer J Clin. 2021;71(3):209-49.

2. Jeon J, Du M, Schoen RE, Hoffmeister M, Newcomb PA, Berndt SI, et al. Determining Risk of Colorectal Cancer and Starting Age of Screening Based on Lifestyle, Environmental, and Genetic Factors. Gastroenterology. 2018;154(8):2152-64.e19.

3. Xie L, Gao S, Alcaire SM, Aoyagi K, Wang Y, Griffin JK, et al. NLF-1 delivers a sodium leak channel to regulate neuronal excitability and modulate rhythmic locomotion. Neuron. 2013;77(6):1069-82.

4. Kycia I, Wolford BN, Huyghe JR, Fuchsberger C, Vadlamudi S, Kursawe R, et al. A Common Type 2 Diabetes Risk Variant Potentiates Activity of an Evolutionarily Conserved Islet Stretch Enhancer and Increases C2CD4A and C2CD4B Expression. Am J Hum Genet, 2018;102(4):620-35.

5. Kuo T, Kraakman MJ, Damle M, Gill R, Lazar MA, Accili D. Identification of C2CD4A as a human diabetes susceptibility gene with a role in $\beta$ cell insulin secretion. Proc Natl Acad Sci U S A. 2019;1 16(40):20033-42.

6. Kaiser AM, Attardi LD. Deconstructing networks of p53-mediated tumor suppression in vivo. Cell Death Differ. 2018;25(1):93-103. 
7. Oren M. Decision making by p53: life, death and cancer. Cell Death Differ. 2003;10(4):431-42.

8. Kubbutat $\mathrm{MH}$, Jones $\mathrm{SN}$, Vousden $\mathrm{KH}$. Regulation of p53 stability by Mdm2. Nature. 1997;387(6630):299-303.

9. Memczak S, Jens M, Elefsinioti A, Torti F, Krueger J, Rybak A, et al. Circular RNAs are a large class of animal RNAs with regulatory potency. Nature. 2013;495(7441):333-8.

10. Vo JN, Cieslik M, Zhang Y, Shukla S, Xiao L, Zhang Y, et al. The Landscape of Circular RNA in Cancer. Cell. 2019;176(4):869-81.e13.

11. Pamudurti NR, Bartok $O$, Jens M, Ashwal-Fluss R, Stottmeister C, Ruhe L, et al. Translation of CircRNAs. Mol Cell. 2017;66(1):9-21.e7.

12. Barrett SP, Salzman J. Circular RNAs: analysis, expression and potential functions. Development. 2016;143(11):1838-47.

13. Zhu Z, Rong Z, Luo Z, Yu Z, Zhang J, Qiu Z, et al. Circular RNA circNHSL1 promotes gastric cancer progression through the miR-1306-3p/SIX1/ vimentin axis. Mol Cancer. 2019;18(1):126.

14. Xie F, Li Y, Wang M, Huang C, Tao D, Zheng F, et al. Circular RNA BCRC-3 suppresses bladder cancer proliferation through miR-182-5p/p27 axis. Mol Cancer. 2018;17(1):144.

15. Liang WC, Wong CW, Liang PP, Shi M, Cao Y, Rao ST, et al. Translation of the circular RNA circ $\beta$-catenin promotes liver cancer cell growth through activation of the Wnt pathway. Genome Biol. 2019;20(1):84

16. Jian X, He H, Zhu J, Zhang Q, Zheng Z, Liang X, et al. Hsa_circ_001680 affects the proliferation and migration of CRC and mediates its chemoresistance by regulating BMl1 through miR-340. Mol Cancer. 2020;19(1):20.

17. Luo Z, Rong Z, Zhang J, Zhu Z, Yu Z, Li T, et al. Circular RNA circCCDC9 acts as a miR-6792-3p sponge to suppress the progression of gastric cancer through regulating CAV1 expression. Mol Cancer. 2020;19(1):86.

18. Hu W, Chen S, Thorne RF, Wu M. TP53, TP53 Target Genes (DRAM, TIGAR), and Autophagy. Adv Exp Med Biol. 2019;1206:127-49.

19. Cordani M, Butera G, Dando I, Torrens-Mas M, Butturini E, Pacchiana $R$, et al. Mutant p53 blocks SESN1/AMPK/PGC-1alpha/UCP2 axis increasing mitochondrial O2-. production in cancer cells. Br J Cancer. 2018;119(8):994-1008

20. Yue X, Zhao Y, Xu Y, Zheng M, Feng Z, Hu W. Mutant p53 in Cancer: Accumulation, Gain-of-Function, and Therapy. J Mol Biol. 2017:429(11):1595-606

21. Shamseddine AA, Clarke CJ, Carroll B, Airola MV, Mohammed S, Rella A, et al. P53-dependent upregulation of neutral sphingomyelinase-2: role in doxorubicin-induced growth arrest. Cell Death Dis. 2015;6:e1947.

22. Ciechanover A. The ubiquitin-proteasome proteolytic pathway. Cell. 1994;79(1):13-21.

23. Chao CC. Mechanisms of p53 degradation. Clin Chim Acta. 2015;438:139-47.

24. Oliner JD, Pietenpol JA, Thiagalingam S, Gyuris J, Kinzler KW, Vogelstein B. Oncoprotein MDM2 conceals the activation domain of tumour suppressor p53. Nature. 1993;362(6423):857-60.

25. Karni-Schmidt O, Lokshin M, Prives C. The Roles of MDM2 and MDMX in Cancer. Annu Rev Pathol. 2016:11:617-44.

26. Lee YS, Dutta A. MicroRNAs in cancer. Annu Rev Pathol. 2009;4:199-227.

27. Yan D, Dong W, He Q, Yang M, Huang L, Kong J, et al. Circular RNA circPICALM sponges miR-1265 to inhibit bladder cancer metastasis and influence FAK phosphorylation. EBioMedicine. 2019:48:316-31.

28. Pan G, Hu T, Chen X, Zhang C. Upregulation Of circMMP9 Promotes Osteosarcoma Progression Via Targeting miR-1265/CHI3L1 Axis. Cancer Manag Res. 2019;11:9225-31.

29. Weber CE, Luo C, Hotz-Wagenblatt A, Gardyan A, Kordass T, Holland-Letz T, et al. miR-339-3p Is a Tumor Suppressor in Melanoma. Cancer Res. 2016;76(12):3562-71.
30. Ma F, Zhang L, Ma L, Zhang Y, Zhang J, Guo B. MiR-361-5p inhibits glycolytic metabolism, proliferation and invasion of breast cancer by targeting FGFR1 and MMP-1. J Exp Clin Cancer Res. 2017;36(1):158.

31. Ma F, Song H, Guo B, Zhang Y, Zheng Y, Lin C, et al. MiR-361-5p inhibits colorectal and gastric cancer growth and metastasis by targeting staphylococcal nuclease domain containing-1. Oncotarget. 2015;6(19):17404-16.

32. Zhou C, LU Y, Li X. miR-339-3p inhibits proliferation and metastasis of colorectal cancer. Oncol Lett. 2015;10(5):2842-8.

33. Gao F, Du Y, Zhang Y, Ren D, Xu J, Chen D. Circ-EZH2 knockdown reverses DDAH1 and CBX3-mediated cell growth and invasion in glioma through miR-1265 sponge activity. Gene. 2020;726:144196.

34. Zhong Y, Du Y, Yang X, Mo Y, Fan C, Xiong F, et al. Circular RNAs function as ceRNAs to regulate and control human cancer progression. Mol Cancer. 2018;17(1):79.

35. Deng G, Mou T, He J, Chen D, Lv D, Liu H, et al. Circular RNA circRHOBTB3 acts as a sponge for miR-654-3p inhibiting gastric cancer growth. J Exp Clin Cancer Res. 2020;39(1):1.

36. Hong X, Liu N, Liang Y, He Q, Yang X, Lei Y, et al. Circular RNA CRIM1 functions as a ceRNA to promote nasopharyngeal carcinoma metastasis and docetaxel chemoresistance through upregulating FOXQ1. Mol Cancer. 2020;19(1):33.

37. Wade M, Li YC, Wahl GM. MDM2, MDMX and p53 in oncogenesis and cancer therapy. Nat Rev Cancer. 2013;13(2):83-96.

38. Huang C, Wu S, Ji H, Yan X, Xie Y, Murai S, et al. Identification of XBP1-u as a novel regulator of the MDM2/p53 axis using an shRNA library. Sci Adv. 2017;3(10):e1701383.

39. Wang S, Zhang Y, Huang J, Wong CC, Zhai J, Li C, et al. TRIM67 Activates p53 to Suppress Colorectal Cancer Initiation and Progression. Cancer Res. 2019;79(16):4086-98.

40. Ni T, Li XY, Lu N, An T, Liu ZP, Fu R, et al. Snail1-dependent p53 repression regulates expansion and activity of tumour-initiating cells in breast cancer. Nat Cell Biol. 2016;18(11):1221-32.

41. Liu X, Meng X, Peng X, Yao Q, Zhu F, Ding Z, et al. Impaired AGO2/miR185-3p/NRP1 axis promotes colorectal cancer metastasis. Cell Death Dis. 2021;12(4):390.

42. Long F, Lin Z, Li L, Ma M, Lu Z, Jing L, et al. Comprehensive landscape and future perspectives of circular RNAs in colorectal cancer. Mol Cancer. 2021;20(1):26.

43. Kristensen LS, Hansen TB, Venø MT, Kjems J. Circular RNAs in cancer: opportunities and challenges in the field. Oncogene. 2018;37(5):555-65.

44. Zhu Z, Yu Z, Rong Z, Luo Z, Zhang J, Qiu Z, et al. The novel GINS4 axis promotes gastric cancer growth and progression by activating Rac 1 and CDC42. Theranostics. 2019:9(26):8294-311.

45. Yang H, Li X, Meng Q, Sun H, Wu S, Hu W, et al. CircPTK2 (hsa_ circ_0005273) as a novel therapeutic target for metastatic colorectal cancer. Mol Cancer. 2020;19(1):13.

46. Huang WJ, Wang Y, Liu S, Yang J, Guo SX, Wang L, et al. Silencing circular RNA hsa_circ_0000977 suppresses pancreatic ductal adenocarcinoma progression by stimulating miR-874-3p and inhibiting PLK1 expression. Cancer Lett. 2018:422:70-80.

47. Yang C, Yuan W, Yang X, Li P, Wang J, Han J, et al. Circular RNA circ-ITCH inhibits bladder cancer progression by sponging miR-17/miR-224 and regulating p21. PTEN expression. Mol Cancer. 2018;17(1):19.

\section{Publisher's Note}

Springer Nature remains neutral with regard to jurisdictional claims in published maps and institutional affiliations. 